\title{
Relative contribution of type 1 and type 2 diabetes loci to the genetic etiology of adult-onset, non-insulin-requiring autoimmune diabetes
}

Rajashree Mishra ${ }^{1 \dagger}$, Alessandra Chesi ${ }^{1 \dagger}$, Diana L. Cousminer ${ }^{2+}$, Mohammad I. Hawa $^{3}$, Jonathan P. Bradfield ${ }^{4}$, Kenyaita M. Hodge ${ }^{1}$, Vanessa C. Guy ${ }^{1}$, Hakon Hakonarson 1,4,5, Bone Mineral Density in Childhood Study, Didac Mauricio ${ }^{6}$, Nanette C. Schloot ${ }^{7}$, Knud B. Yderstræde ${ }^{8}$, Benjamin F. Voight ${ }^{2,9}$, Stanley Schwartz ${ }^{10}$, Bernhard O. Boehm ${ }^{11,12+}$, Richard David Leslie $e^{3,14^{*}+}$, and Struan F. A. Grant ${ }^{1,2,4,5,13^{*}+}$

\begin{abstract}
Background: In adulthood, autoimmune diabetes can present as non-insulin-requiring diabetes, termed as 'latent autoimmune diabetes in adults' (LADA). In this study, we investigated established type 1 diabetes (T1D) and type 2 diabetes (T2D) genetic loci in a large cohort of LADA cases to assess where LADA is situated relative to these two well-characterized, classic forms of diabetes.

Methods: We tested the association of T1D and T2D GWAS-implicated loci in 978 LADA cases and 1057 nondiabetic controls of European ancestry using a linear mixed model. We then compared the associations of T1D and T2D loci between LADA and T1D and T2D cases, respectively. We quantified the difference in genetic risk between each given disease at each locus, and also calculated genetic risk scores to quantify how genetic liability to T1D and T2D distinguished LADA cases from controls.
\end{abstract}

Results: Overall, our results showed that LADA is genetically more similar to T1D, with the exception of an association at the T2D HNF1A locus. Several T1D loci were associated with LADA, including the major histocompatibility complex region, as well as at PTPN22, SH2B3, and INS. Contrary to previous studies, the key T2D risk allele at TCF7L2 (rs7903146-T) had a significantly lower frequency in LADA cases, suggesting that this locus does not play a role in LADA etiology. When constrained on antibody status, the similarity between LADA and T1D became more apparent; however, the HNF1A and TCF7L2 observations persisted.

Conclusion: LADA is genetically closer to T1D than T2D, although the genetic load of T1D risk alleles is less than childhood-onset T1D, particularly at the major histocompatibility complex region, potentially accounting for the later disease onset. Our results show that the genetic spectrum of T1D extends into adult-onset diabetes, where it can clinically masquerade as T2D. Furthermore, T2D genetic risk plays a small role in LADA, with a degree of evidence for the HNF1A locus, highlighting the potential for genetic risk scores to contribute towards defining diabetes subtypes.

Keywords: Latent autoimmune diabetes in adults, Genetic risk scores

\footnotetext{
* Correspondence: r.d.g.leslie@qmul.ac.uk; grants@chop.edu

${ }^{\dagger}$ Equal contributors

${ }^{3}$ Department of Immunobiology, Barts and the London School of Medicine

and Dentistry, Queen Mary University of London, London, UK

'Division of Human Genetics, The Children's Hospital of Philadelphia,

Philadelphia, PA, USA

Full list of author information is available at the end of the article
} 


\section{Background}

Diabetes is a heterogeneous group of diseases resulting in hyperglycemia due to insulin secretory dysfunction as well as insulin resistance. A substantial proportion of type 1 diabetes (T1D) cases present in adulthood, and despite the presence of diabetes-associated autoantibodies, the majority of these patients do not initially require insulin $[1,2]$. The manifestation of this 'latent autoimmune diabetes in adulthood' (LADA) is clinically defined by (1) an adult age of onset, (2) at least one diabetes-associated autoantibody, and (3) the lack of requisite insulin treatment for at least 6 months after diagnosis. This definition overall represents approximately $5-10 \%$ of all cases of adult-onset diabetes, potentially the most frequent form of autoimmune diabetes $[3,4]$.

However, classifying adult-onset autoimmune T1D, including LADA, remains challenging. The need for insulin treatment is a clinical decision, while diabetesassociated autoantibodies are neither pathogenic nor categorical features of LADA. Decisions are further confounded by false positives when large numbers of patients are screened [5]. Since LADA has intermediate features between T1D and type 2 diabetes (T2D), there are limits to the current classification of diabetes. New paradigms are needed to distinguish LADA and ensure appropriate disease treatment and management.

Recently, several studies have used genetic information derived from diabetes-associated risk variants across the genome to reclassify diabetes [6]. To date, comprehensive genetic studies of T1D and T2D have uncovered dozens of distinct susceptibility loci for each of these two diseases [7-9]. Initial analyses of T1D loci in relatively small LADA cohorts have consistently shown an association with the T1D locus HLA-DQB1, which resides in the major histocompatibility complex (MHC) $[3,10,11]$, as well as at PTPN22 and INS $[12,13]$. Similar analyses of T2D loci have suggested an association in LADA with the strongest T2D locus harboring TCF7L2 [12, 14, 15] and the ZMIZ1 locus [16]. A significant challenge of these studies has been the lack of statistical power due to the small number of LADA patients included. Thus, the genetic etiology of LADA remains largely unresolved.

To quantify the genetic liability to LADA contributed by genetic risk factors for $\mathrm{T} 1 \mathrm{D}$ and $\mathrm{T} 2 \mathrm{D}$, we amassed the largest LADA cohort to date. By assessing the association of these variants in LADA, our objective was to place LADA along the etiological diabetes spectrum and reshape our understanding of the relationship between LADA and classic diabetes phenotypes.

\section{Methods}

Study populations and antibody testing

We ascertained 978 LADA cases from two studies, a European Union-funded multicenter study (Action
LADA) and a German Research Council study (DFG: SFB 518, A1), each of which aimed to identify features of adult-onset autoimmune diabetes. A description of the participants and study design has been published elsewhere [2]. For this particular study, the criteria for LADA diagnosis was more strict to avoid potential false positives. All participants were diagnosed with LADA if they were aged 30-70 years, tested positive for diabetesassociated glutamic acid decarboxylase autoantibodies (GADA), and were not given insulin treatment for at least 6 months after diagnosis. Samples were tested for serum autoantibodies to GADA and insulinomaassociated antigen-2 (IA2A) (Additional file 1: Supplemental Methods, which also includes all genotyping methods and quality control).

The population-based control cohort comprised 1057 non-diabetic children of European ancestry, aged 5-20 years, enrolled in the Bone Mineral Density in Childhood Study (BMDCS). Subjects were randomly recruited from five different centers in the USA. As previously reported [17], enrollment criteria included healthy, normally developing children. Each participating center received approval of the study by their respective institutional review boards.

Since BMDCS consists of European-descent children ascertained from the USA, while the LADA cases were adults ascertained from the UK and Germany, we also leveraged 2820 healthy adult British birth cohort controls from the Wellcome Trust Case Control Consortium (WTCCC) [8] to act as an extra set of controls to verify our observations. Principal component analysis (PCA) showed that BMDCS controls were well-matched with cases despite ascertainment in the USA, while the WTCCC controls were stratified (Additional file 1: Figure S1) principally due to differences in the genotyping arrays used. Thus, BMDCS was used in the primary analyses, with verification in the WTCCC cohort. Our study also utilized publicly available childhood-onset T1D ( $\mathrm{n}=2000)$ and adult-onset T2D ( $\mathrm{n}=1999)$ Affymetrix $500 \mathrm{~K}$ genotype data from the WTCCC; these individuals were recruited within England, Scotland, and Wales [8]. Individual data from WTCCC is available through the Consortium's Data access committee (http://www.wtccc.org.uk). The genomic inflation factor for the pruned genome-wide SNPs is 0.966 and the QQplot can be found in Additional file 1: Figure S2.

\section{Individual candidate SNP association tests}

To investigate the role of previously discovered T1D and T2D variants in LADA, we tested 67 T1D SNPs (from Immunobase; http://www.immunobase.org, and 71 T2D SNPs from the T2D study led by the DIAbetes Genetics Replication And Meta-analysis (DIAGRAM) Consortium [9]). Association between each SNP and case/control 
status was assessed using a univariate linear mixed model within GEMMA [18]. This model accounts for population stratification and relatedness using the Wald test and the restricted maximum likelihood estimate of $\beta$. We tested each SNP in LADA cases versus BMDCS controls and in LADA cases versus T1D or T2D cases. Significant associations were called after Bonferroni correction for multiple testing. Analysis was performed for all LADA cases $(n=978)$, LADA cases positive for GADA only $(n=669)$, and LADA cases positive for both GADA and IA2A $(n=309)$. Approximated odds ratios were calculated using $\mu$ (intercept) and $\beta$ (effect size) estimates from the linear mixed model, with the formula: $\mathrm{OR}=\frac{\beta}{e^{\mu(1-\mu)}}[18]$.

\section{Genetic risk scores (GRS)}

We calculated two GRS using 69 T1D and T2D SNPs for T1D cases $(n=1990)$, T2D cases $(n=1960)$, LADA cases $(n=978)$, LADA cases positive for GADA only ( $n$ = 669), LADA cases restricted on GADA + IA2A+ status $(\mathrm{n}=309)$, and BMDCS controls $(\mathrm{n}=1057)$. Weights utilized for the scores were derived from published odds ratios (ORs) from $\mathrm{T} 1 \mathrm{Db}$ ase (t1dbase.org) or a previous publication [19], respectively. Two SNPs, rs2187668 and rs7454108, were used to infer HLA DR3/DR4/DQ8 haplotypes, and additional HLA SNPs tagging HLA A, HLA $B$, and DRB1 haplotypes were included $[6,20]$. rs7111341 and rs11171710 did not have publicly available ORs, and rs7202877 is implicated in both T1D and T2D (Additional file 1: Table S1), so these were excluded. Each GRS was calculated using PLINK by multiplying the number of risk-increasing alleles by the natural log of the OR at each locus and summing across risk loci for each individual. Logistic regression and receiver operating characteristic (ROC) curve analyses evaluated how well these GRS distinguished LADA cases from BMDCS controls (using the PredictABEL package
[21]). We repeated the GRS calculation for GADA+ and IA2+ LADA cases and for GADA+, IA2A- LADA cases. Additionally, we combined the T1D and T2D SNPs (139 SNPs) and classified LADA and controls for both LADA groups. The distributions of the T1D and T2D GRS of the five groups were compared using the Wilcoxon rank sum test accounting for multiple comparisons (using a Bonferroni correction). Control samples were obtained from the WTCCC2 study, as described above.

\section{Results}

\section{T1D loci}

Four T1D SNPs were significantly associated with LADA and survived multiple testing correction $(P=0.05 / 67$, loci tested $=7.46 \times 10^{-4}$; Table 1 and Additional file 1 : Table S2). The strongest association was at the MHC region $\left(\mathrm{OR}=1.46 ; P=9.64 \times 10^{-11}\right)$. Strong association was also observed for variants at PTPN22 $(\mathrm{OR}=1.47 ; P=$ $\left.6.38 \times 10^{-6}\right), S H 2 B 3\left(\mathrm{OR}=1.28 ; P=1.10 \times 10^{-5}\right)$, and INS $\left(\mathrm{OR}=1.27 ; \quad P=2.39 \times 10^{-4}\right)$. The association signal within the $\mathrm{MHC}$ region was significantly different between LADA and T1D cases $\left(P_{\text {difference }}=1.26 \times 10^{-17}\right)$, with the T1D risk allele of rs9272346 (A) less common in LADA than in T1D, but still at a higher frequency than in controls. The signals at INS and SMARCE1 also yielded significant differences between LADA and T1D $\left(P_{\text {difference }}=3.88 \times 10^{-4}\right.$ and $6.54 \times 10^{-4}$, respectively $)$. The INS signal was more common in LADA than in either T1D or controls, while the frequency of the SMARCE1 signal was lower in LADA than in T1D but similar to controls.

To further understand the influence of antibody status on the clinical classification of LADA, the same analyses were carried out for 669 GADA+ LADA subjects (Additional file 1: Table S3). The MHC region was the only signal surviving correction for multiple comparison for cases against controls, as well as cases versus T1D $\left(\mathrm{OR}=1.30 ; P=6.84 \times 10^{-5}, P_{\text {difference }}=1.99 \times 10^{-24}\right)$.

Table 1 Association of established type 1 diabetes (T1D) loci with latent autoimmune diabetes in adulthood (LADA). Only T1D variants significantly associated with LADA are shown (LADA association $P$ value), as well as signals significantly different between LADA and T1D (LADA vs. T1D $P$ value), with a significance threshold of $P=7.46 \times 10^{-4}$. The locus reported is the closest gene of interest to the signal (a full list of genes is provided in Additional file 1: Table S2). The risk and other alleles reported refer to the alleles in T1D, and the following allele frequencies refer to the frequency of the risk allele reported in T1D for LADA, T1D, and Bone Mineral Density in Childhood Study (BMDCS) control group. Odds ratios of the risk allele reported are derived from the BMDCS control data set $(n=1057)$, the Wellcome Trust Case Control Consortium T1D $(n=1990)$, and the LADA cases $(n=978)$

\begin{tabular}{|c|c|c|c|c|c|c|c|c|}
\hline \multirow[t]{2}{*}{ Locus } & \multirow[t]{2}{*}{ SNP } & \multirow{2}{*}{$\begin{array}{l}\text { T1D alleles } \\
\text { risk/other }\end{array}$} & \multicolumn{3}{|c|}{ Risk allele frequency } & \multirow[t]{2}{*}{ LADA odds ratio } & \multirow{2}{*}{$\begin{array}{l}\text { LADA } P \\
\text { value }\end{array}$} & \multirow{2}{*}{$\begin{array}{l}\text { LADA vs. T1D } \\
P \text { value }\end{array}$} \\
\hline & & & LADA & $\mathrm{T} 1 \mathrm{D}$ & Control & & & \\
\hline$\overline{M H C}$ & rs9272346 & $A / G$ & 0.686 & 0.818 & 0.579 & $1.455(1.427-1.483)$ & $9.6 \times 10^{-11}$ & $1.26 \times 10^{-17}$ \\
\hline PTPN22 & rs6679677 & $\mathrm{A} / \mathrm{C}$ & 0.143 & 0.17 & 0.093 & $1.469(1.427-1.510)$ & $6.38 \times 10^{-6}$ & $2.61 \times 10^{-2}$ \\
\hline $\mathrm{SH} 2 \mathrm{~B} 3$ & rs17696736 & $\mathrm{G} / \mathrm{A}$ & 0.515 & 0.503 & 0.44 & $1.277(1.250-1.304)$ & $1.10 \times 10^{-5}$ & 0.542 \\
\hline INS & rs689 & $\mathrm{T} / \mathrm{A}$ & 0.796 & 0.741 & 0.73 & $1.265(1.234-1.296)$ & $2.39 \times 10^{-4}$ & $3.88 \times 10^{-4}$ \\
\hline SMARCE1 & rs7221109 & $C / T$ & 0.621 & 0.687 & 0.632 & $0.954(0.925-0.983)$ & 0.423 & $6.54 \times 10^{-4}$ \\
\hline
\end{tabular}


In the restricted subset of GADA+ IA2A+ LADA cases $(\mathrm{n}=309)$, four loci were associated (Table 2 and Additional file 1: Table S4). The MHC $(\mathrm{OR}=1.98 ; P=1.20 \times$ $\left.10^{-15}\right)$, PTPN22 (OR $\left.=1.86 ; P=2.19 \times 10^{-6}\right), S H 2 B 3(\mathrm{OR}$ $\left.=1.48 ; P=5.93 \times 10^{-6}\right)$, and INS (rs689; OR=1.44; $P=$ $1.90 \times 10^{-4}$ ) signals remained strongly associated and had stronger ORs in this constrained setting. However, the risk-increasing allele at the MHC locus remained significantly less than that in T1D cases. Two partially independent signals near INS $\left(\mathrm{r}^{2}=0.278\right)$ yielded a significant difference between T1D and GADA+ IA2A+ LADA in this restricted dataset, $r 5689\left(P_{\text {difference }}=1.68 \times\right.$ $\left.10^{-6}\right)$ and rs7111341 $\left(P_{\text {difference }}=2.39 \times 10^{-4}\right)$.

\section{T2D loci}

Only one T2D signal survived correction for multiple comparisons $\left(P=0.05 / 71\right.$, loci $\left.=7.04 \times 10^{-4}\right)$ in LADA cases, the HNF1A locus (OR $=1.291 ; P=3.42 \times 10^{-4}$; Table 3 and Additional file 1: Table S5). Contrary to previous reports $[12,14,15]$, the T2D risk allele (rs7903146-T) at TCF7L2 was not enriched among LADA cases, with a frequency close to that of controls (0.295 vs. 0.298 , respectively); indeed, the TCF7L2 signal was the most significantly different signal between LADA and T2D cases $\left(P_{\text {difference }}=5.21 \times 10^{-6}\right)$. In the GADA+ restricted set, there were no association signals surviving correction for multiple comparisons, and the only signal showing a significant difference between LADA and T2D was the depletion of the TCF7L2 $\mathrm{T}$ allele $\left(P_{\text {difference }}=5.03 \times 10^{-4}\right.$; Additional file 1: Table S6), where the $\mathrm{T}$ allele showed modest, albeit non-significant excess when compared to controls $(\mathrm{OR}=1.088)$.

In the restricted set of 309 GADA+ IA2A+ LADA subjects, HNF1A continued to yield a significant association $\left(\mathrm{OR}=1.47 ; P=2.52 \times 10^{-4}\right.$; Table 4 and Additional file 1: Table S7). Again, the TCF7L2 locus was significantly different between LADA and T2D cases $\left(P_{\text {difference }}=2.56 \times 10^{-7}\right)$, with the risk allele frequency even less than that in controls in this restricted case set (allele frequency of 0.251 vs. 0.298 in LADA and controls, respectively).

Leveraging 2820 healthy adult British subjects from the WTCCC as alternative controls, we observed very consistent results overall (Additional file 1: Table S8 and Supplementary Results) despite the array differences for this set.

\section{GRS}

A high T1D GRS implies a high genetic risk for that disease. Figure 1 shows that the T1D GRS better predicted whether a subject is a LADA case or control than the T2D GRS. The areas under the curve (AUC) for the T1D and T2D GRS were 0.667 and 0.565 , respectively (Fig. 1a). Thus, when considering adult-onset diabetes patients who do not initially require insulin, genetic risk defined for T1D could better identify autoimmune diabetes cases than genetic risk defined for T2D.

This result was more pronounced when considering controls versus 309 GADA+ IA2A+ LADA cases (Fig. 1b) (AUC for T1D GRS $=0.760$, T2D GRS $=0.496$ ). However, these results were less pronounced for the 669 GADA+ only LADA cases versus controls (AUC for T1D GRS $=0.623$, T2D GRS $=0.597$ ). The combined effect of genetic risk using both T1D and T2D SNPs marginally improved classification of LADA cases and controls (AUC $=0.673$ ) and classification of GADA+ LADA and controls (AUC $=0.635$ ). However, there was no improvement of classification between GADA+ IA2A + LADA and controls (AUC $=0.755$ ) using a combination of T1D-T2D SNPs. To highlight the important role of non-HLA loci in discrimination, we calculated T1D GRS without the HLA region and an HLA only GRS.

Table 2 Association of established type 1 diabetes (T1D) loci in latent autoimmune diabetes in adulthood (LADA) subjects positive for both glutamic acid decarboxylase autoantibodies and insulinoma-associated antigen-2 autoantibodies. Only T1D variants significantly associated with LADA are shown (LADA association $P$ value), as well as signals significantly different between LADA and T1D (LADA vs. T1D $P$ value). Significance threshold is $7.46 \times 10^{-4}$ after correcting for multiple comparison. The locus reported is the closest, well-known gene of interest to the signal (a full list of genes is provided in Additional file 1: Table S3). The risk and other alleles reported refer to the alleles in T1D, and the following allele frequencies refer to the frequency of the risk allele reported in T1D for LADA, T1D, and Bone Mineral Density in Childhood Study (BMDCS) control group. Odds ratios of the risk allele reported are derived from the BMDCS control data set $(n=1057)$, the Wellcome Trust Case Control Consortium T1D $(n=1990)$, and the constrained N LADA cases ( $n=309)$. *Independent signals (INS signals have an $r^{2}=0.278$ )

\begin{tabular}{|c|c|c|c|c|c|c|c|c|}
\hline \multirow[t]{2}{*}{ Locus } & \multirow[t]{2}{*}{ SNP } & \multirow{2}{*}{$\begin{array}{l}\text { T1D alleles } \\
\text { risk/other }\end{array}$} & \multicolumn{3}{|c|}{ Risk allele frequency } & \multirow[t]{2}{*}{ LADA odds ratio } & \multirow{2}{*}{$\begin{array}{l}\text { LADA P } \\
\text { value }\end{array}$} & \multirow{2}{*}{$\begin{array}{l}\text { LADA vs. T1D } \\
P \text { value }\end{array}$} \\
\hline & & & $\overline{L A D A}$ & T1D & $\overline{\text { Control }}$ & & & \\
\hline$\overline{M H C}$ & rs9272346 & $\mathrm{A} / \mathrm{G}$ & 0.763 & 0.818 & 0.579 & $1.983(1.954-2.012)$ & $1.20 \times 10^{-15}$ & $4.01 \times 10^{-3}$ \\
\hline PTPN22 & rs6679677 & $\mathrm{A} / \mathrm{C}$ & 0.17 & 0.17 & 0.093 & $1.864(1.819-1.909)$ & $2.19 \times 10^{-6}$ & 0.603 \\
\hline $\mathrm{SH} 2 \mathrm{~B} 3$ & rs17696736 & $\mathrm{G} / \mathrm{A}$ & 0.542 & 0.503 & 0.44 & $1.481(1.452-1.511)$ & $5.93 \times 10-6$ & 0.180 \\
\hline$I N S^{*}$ & rs689 & $\mathrm{T} / \mathrm{A}$ & 0.824 & 0.741 & 0.73 & $1.440(1.407-1.474)$ & $1.90 \times 10^{-4}$ & $1.68 \times 10^{-6}$ \\
\hline INS $^{*}$ & rs7111341 & $C / T$ & 0.812 & 0.75 & 0.73 & $1.360(1.327-1.394)$ & $1.82 \times 10^{-3}$ & $2.39 \times 10^{-4}$ \\
\hline
\end{tabular}


Table 3 Association of established type 2 diabetes (T2D) loci with latent autoimmune diabetes in adulthood (LADA). Only T2D variants significantly associated with LADA after correcting for multiple comparison $\left(P<7.04 \times 10^{-4}\right)$ are shown (LADA association $P$ value), as well as variants significantly different between LADA and T2D (LADA vs. T2D $P$ value). The locus reported is the closest, well-known gene of interest to the signal (a full list of genes are provided in Additional file 1: Table S4). The risk and other alleles reported refer to the alleles in $T 2 D$, and the following allele frequencies refer to the frequency of the risk allele reported in T2D, for LADA, T2D, and Bone Mineral Density in Childhood Study (BMDCS) control groups. Odds ratios of the risk allele reported are derived from the BMDCS control data set $(n=1057)$, the Wellcome Trust Case Control Consortium T2D $(n=1960)$, and the LADA cases $(n=978)$

\begin{tabular}{|c|c|c|c|c|c|c|c|c|}
\hline \multirow[t]{2}{*}{ Locus } & \multirow[t]{2}{*}{ SNP } & \multirow{2}{*}{$\begin{array}{l}\text { T2D alleles } \\
\text { risk/other }\end{array}$} & \multicolumn{3}{|c|}{ Risk allele frequency } & \multirow[t]{2}{*}{ LADA odds ratio } & \multirow{2}{*}{$\begin{array}{l}\text { LADA P } \\
\text { value }\end{array}$} & \multirow{2}{*}{$\begin{array}{l}\text { LADA vs. T2D } \\
P \text { value }\end{array}$} \\
\hline & & & LADA & $\mathrm{T} 2 \mathrm{D}$ & Control & & & \\
\hline$\overline{H N F 1 A}$ & rs12427353 & $\mathrm{G} / \mathrm{C}$ & 0.831 & 0.828 & 0.787 & $1.291(1.256-1.326)$ & $3.42 \times 10^{-4}$ & 0.538 \\
\hline TCF $72^{\mathrm{a}}$ & rs7903146 & $\mathrm{T} / \mathrm{C}$ & 0.295 & 0.376 & 0.298 & $1.023(0.994-1.053)$ & 0.702 & $5.21 \times 10^{-6}$ \\
\hline
\end{tabular}

${ }^{a}$ Although the control risk allele frequency is greater than the case risk allele frequency, the beta calculated from the linear mixed model is adjusted effects after controlling for population stratification, resulting in an OR slightly above 1

Additionally, we tested these five models of GRS in discrimination between the LADA categories versus T1D and GADA+ only LADA cases versus GADA + IA2A+ LADA cases (Additional file 1: Figure S3). The HLA alone accounted for a strong difference between all LADA cases and T1D cases $(A U C=0.699)$, especially between T1D and GADA+ only LADA cases $(\mathrm{AUC}=$ 0.733). The non-HLA GRS had an AUC of 0.655 for distinguishing GADA + IA2A+ LADA cases from controls. The HLA-only GRS had an AUC of 0.737 for distinguishing GADA + IA2A+ LADA cases from controls, but combining these loci, the AUC was 0.76 .

Comparison of the T1D SNP-GRS distributions among the six groups (T1D, T2D, LADA, GADA+ IA2A+ LADA, GADA-only LADA, and controls; Fig. 2a) revealed significant differences between all pairs $\left(P<10^{-5}\right)$, except T2D versus controls. This observation was as expected as T2D cases should not harbor a high load of T1D risk alleles. Furthermore, there were only nominally significant differences between LADA and GADA-only LADA cases. Of particular note, there was a significant difference in the T1D GRS distribution between T1D and GADA+ IA2A+ LADA, highlighting genetic differences between LADA restricted on IA2A + status and T1D $(P=0.0001)$.
Comparison of the distributions of the T2D SNP-GRS (Fig. 2b) revealed significant differences between LADA and T2D cases $\left(P=3.50 \times 10^{-11}\right)$ and between the GADA+ IA2A+ LADA and T2D cases $\left(P=3.50 \times 10^{-16}\right)$. These results suggest T2D risk alleles are not enriched in LADA, concordant with the results of our single-SNP analyses. However, the T2D SNP-GRS distribution was also significantly different between LADA and T1D cases $\left(P=6.10 \times 10^{-11}\right)$ and controls $\left(P=8.00 \times 10^{-6}\right)$. The T2D risk allele load, although not as high as for T2D, is still higher than that seen in T1D or among the healthy population. We observed a nominally significant difference for T2D risk allele load between GADA+ only LADA and T2D cases $\left(P=5.60 \times 10^{-3}\right)$ and no statistically significant difference between GADA+ only LADA and overall LADA cases.

\section{Discussion}

Defining LADA as a distinct form of T1D has two broad benefits. First, it highlights the potential to understand what determines both the degree and rate of disease progression. Second, it helps define differences between adult-onset autoimmune diabetes, including LADA, and T2D in terms of co-morbidities and putative therapy

Table 4 Association between established type 2 diabetes (T2D) loci in latent autoimmune diabetes in adulthood (LADA) cases positive for glutamic acid decarboxylase autoantibodies (GADA) and insulinoma-associated antigen-2 autoantibodies (IA2A). T2D variants that were significantly associated in LADA cases positive for GAD and IA2 autoantibodies ( $n=309)($ LADA association $P$ value) are shown, as well as signals that were significantly different between LADA and T2D cases (LADA vs. T2D $P$ value). The significance threshold was set to $P<7.04 \times 10^{-4}$ to correct for multiple testing. The locus reported is the closest, well-known gene of interest to the signal (a full list of genes is provided in Additional file 1: Table S5). T2D risk allele frequencies reported are derived from the Bone Mineral Density in Childhood Study control data set ( $n=1057)$, the Wellcome Trust Case Control Consortium T2D $(n=1960)$, and the LADA cases positive for autoantibodies GADA and IA2A $(n=309)$. The odds ratios for LADA are shown both GEMMA-corrected (for relatedness and batch effects) and uncorrected

\begin{tabular}{|c|c|c|c|c|c|c|c|c|}
\hline \multirow[t]{2}{*}{ Locus } & \multirow[t]{2}{*}{ SNP } & \multirow{2}{*}{$\begin{array}{l}\text { T2D alleles } \\
\text { risk/other }\end{array}$} & \multicolumn{3}{|c|}{ Risk allele frequency } & \multirow[t]{2}{*}{ LADA odds ratio } & \multirow{2}{*}{$\begin{array}{l}\text { LADA } P \\
\text { value }\end{array}$} & \multirow{2}{*}{$\begin{array}{l}\text { LADA vs. T2D } \\
P \text { value }\end{array}$} \\
\hline & & & LADA & $\mathrm{T} 2 \mathrm{D}$ & Control & & & \\
\hline HNF1A & rs12427353 & $\mathrm{G} / \mathrm{C}$ & 0.857 & 0.828 & 0.787 & $1.474(1.438-1.511)$ & $2.52 \times 10^{-4}$ & $5.42 \times 10^{-2}$ \\
\hline ZBED3 & rs6878122 & $A / G$ & 0.744 & 0.658 & 0.706 & $1.216(1.184-1.249)$ & $3.86 \times 10^{-2}$ & $1.47 \times 10^{-5}$ \\
\hline TCF7L2 & rs7903146 & $\mathrm{T} / \mathrm{C}$ & 0.251 & 0.376 & 0.298 & $0.852(0.820-0.883)$ & $8.14 \times 10^{-2}$ & $2.56 \times 10^{-7}$ \\
\hline
\end{tabular}



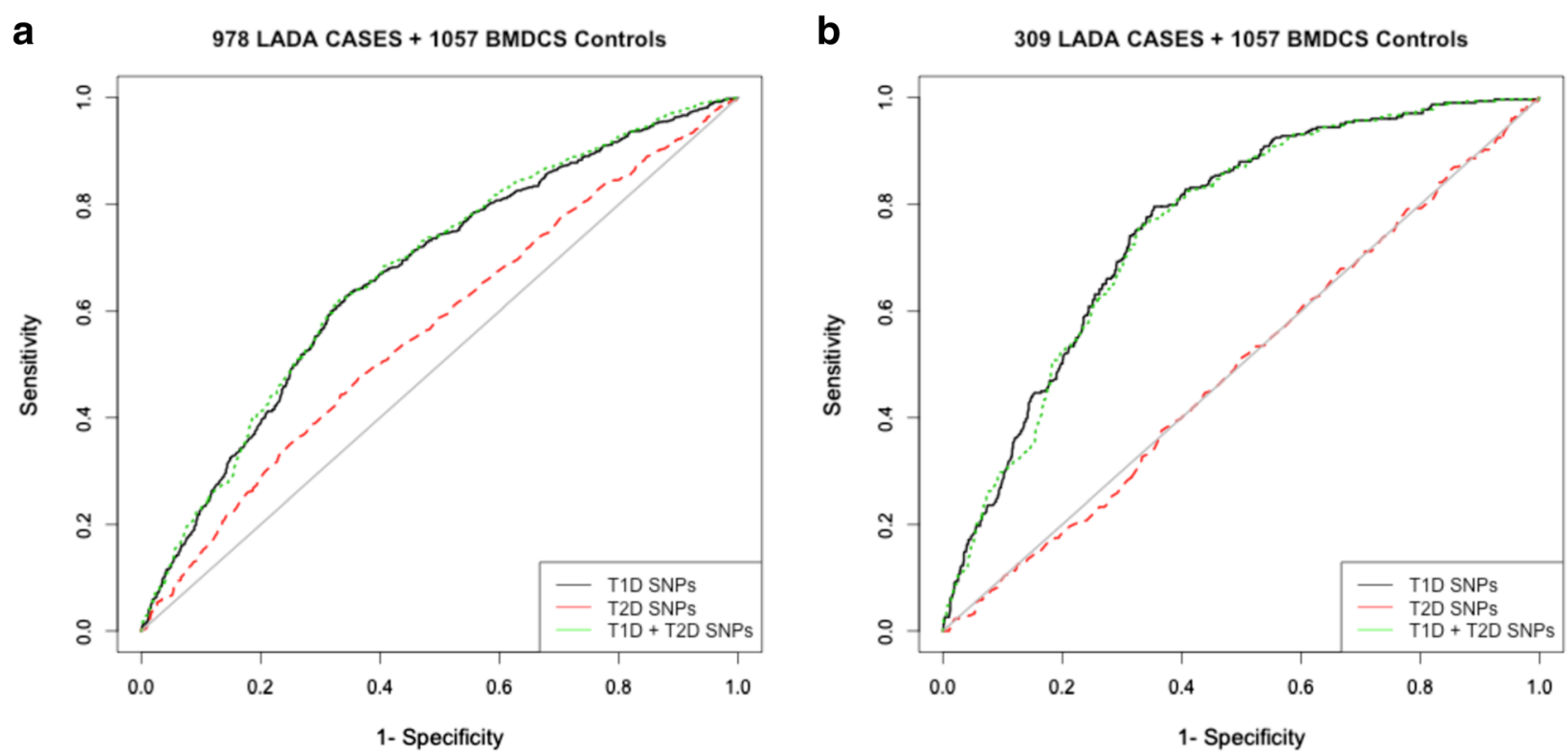

\section{669 LADA CASES + 1057 BMDCS Controls}

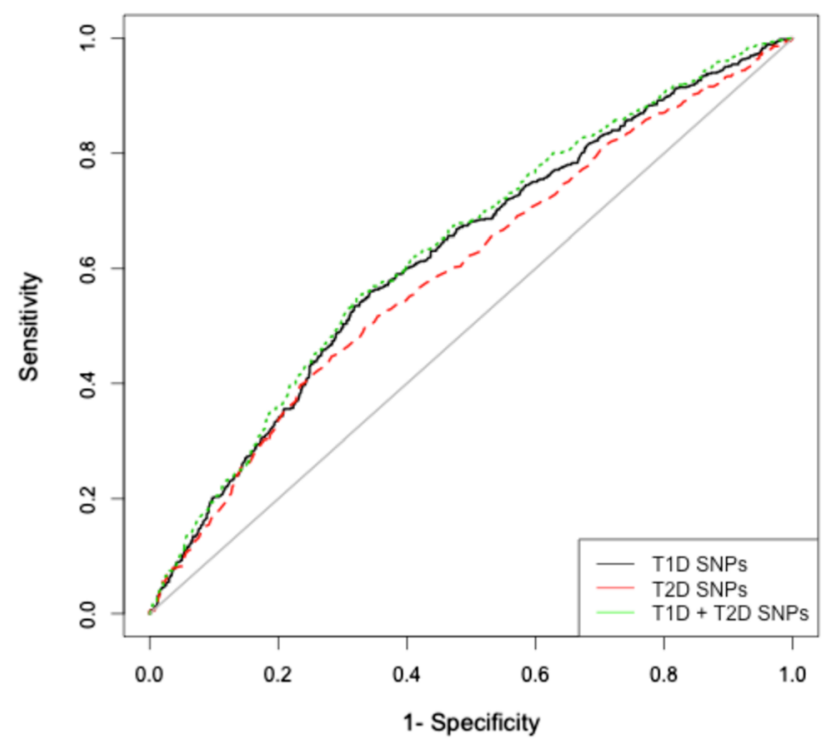

Fig. 1 Type 1 (T1D) and type 2 diabetes (T2D) genetic risk scores (GRS) tested in latent autoimmune diabetes in adulthood (LADA) cases and controls. Weighted GRS for T1D (black) and T2D (red) were calculated by summing over all the risk alleles (T1D/T2D SNPs). The scores were tested in a 978 LADA cases and 1057 healthy controls; b 309 autoantibody-positive (glutamic acid decarboxylase autoantibodies (GADA) and insulinomaassociated antigen-2 autoantibodies (IA2A)) LADA cases and 1057 controls; c 669 GADA-only autoantibody positive. The ability of the GRS to discriminate between cases and controls was assessed by receiver and operator characteristic analysis. The area under the curve (AUC) was 0.667 and 0.565 for T1D and T2D, respectively, in the set with all LADA cases, 0.76 for T1D and 0.496 for T2D in the GADA, IA2A autoantibody-positive restricted set, and 0.623 for T1D, 0.597 for T2D in the GADA-only autoantibody-positive restricted set. A combination of T1D and T2D SNPs (green) had an AUC of 0.673 for all samples, 0.755 for the GADA+ IA2A+ restricted set, and 0.635 for the GADA-only restricted set

[22]. Leveraging children whose future diabetes risk is unknown represents the most conservative setting in which to conduct this study given they serve as excellent population-based controls in which to contrast the cases; however, the conservative nature of the approach may result in some false negative results.
To shed light on the genetic etiology of LADA, we tested the impact of established T1D and T2D risk loci in the largest set of LADA cases collected to date. Our study differs from a previous association study with GWAS-implicated loci in adult-onset autoimmune diabetes by Howson et al. [23]; first, our LADA cases are 
a T1D Genetic Risk Scores

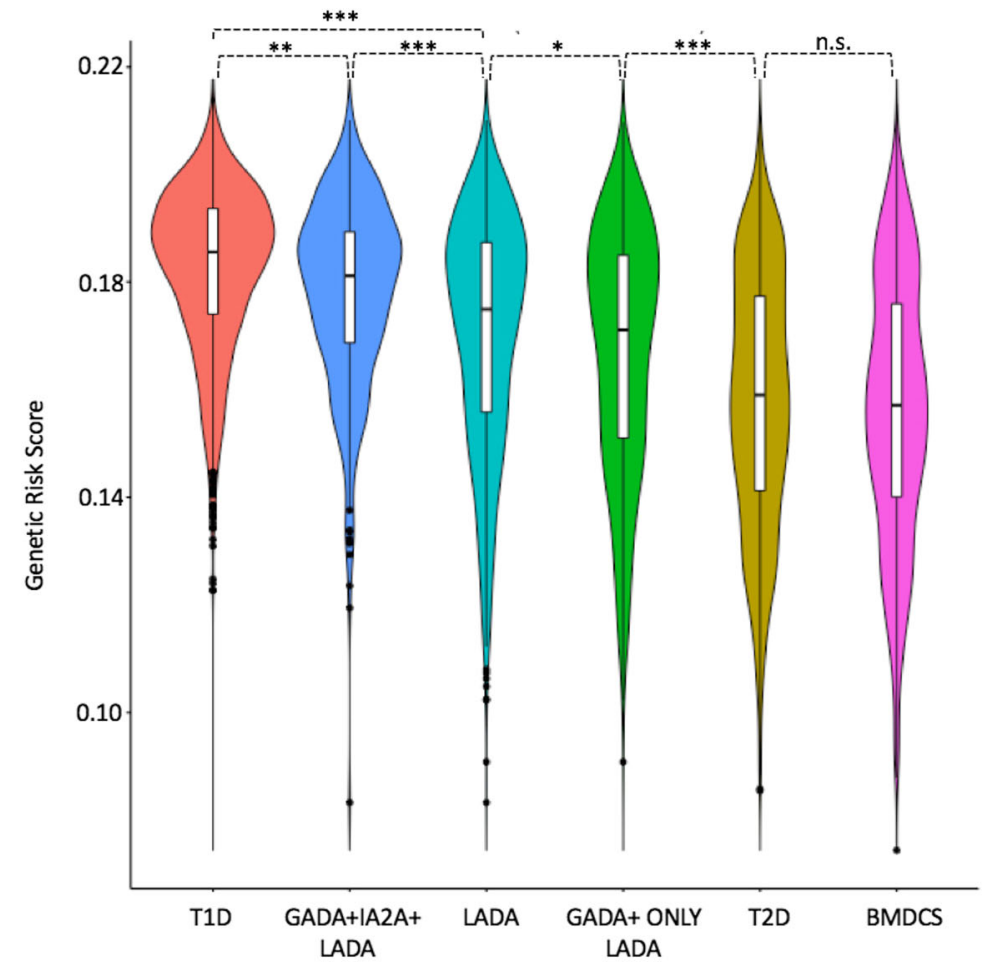

b T2D Genetic Risk Scores

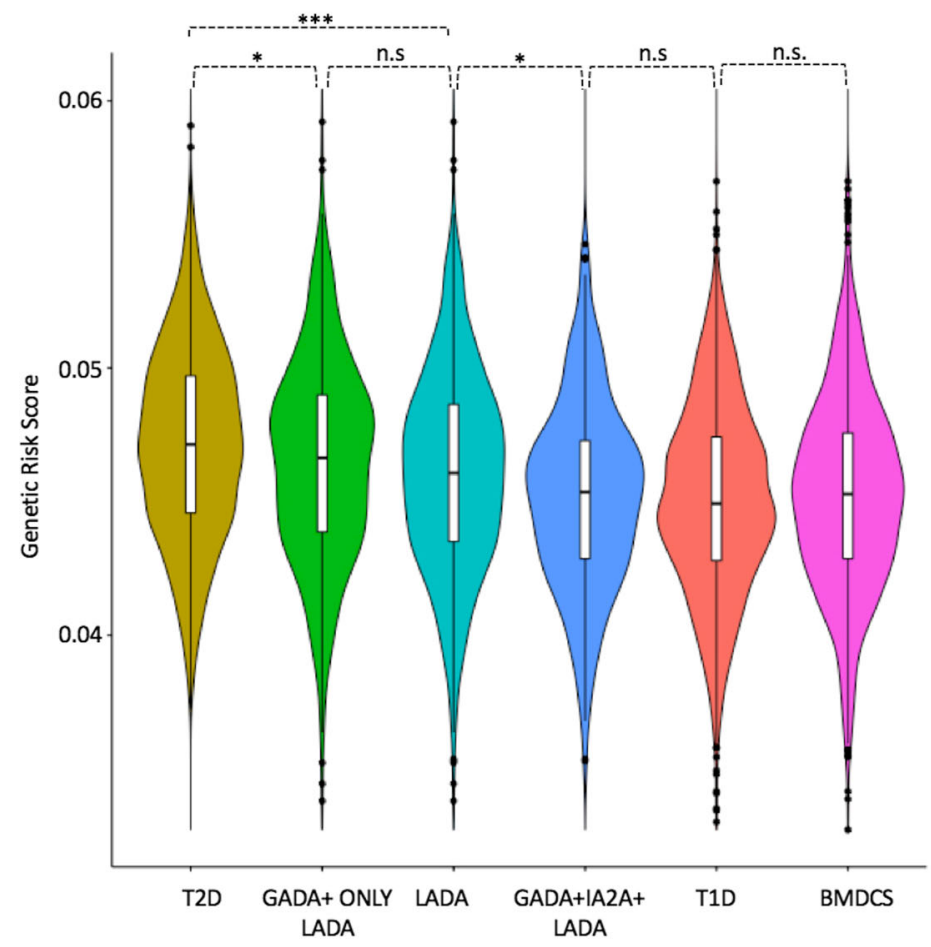

Fig. 2 (See legend on next page.) 
(See figure on previous page.)

Fig. 2 Genetic risk score (GRS) distributions between type 1 diabetes (T1D), type 2 diabetes (T2D), latent autoimmune diabetes in adulthood (LADA), and LADA-restricted cases and controls. The GRS distributions were compared across individuals diagnosed with T1D ( $n=1990)$, T2D $(n=1960)$, LADA ( $n=978)$, LADA restricted $(n=309)$, LADA GADA-only $(n=669)$, and Bone Mineral Density in Childhood Study controls $(n=1057)$. a Violin plots of the distributions of the GRS calculated using the T1D SNPs for the five groups. A multiple comparison test (Wilcoxon rank sum test) was performed to calculate the significance of pair-wise differences. b Violin plots of the distributions of the GRS calculated using the T2D SNPS for the five groups. A multiple comparison test (Wilcoxon rank sum test) was performed to calculate the significance of pair-wise differences. We include some of the significant $P$ values to highlight key differences. $\left({ }^{* * *} P<10^{-5},{ }^{* *} P<0.0001,{ }^{*} P<0.05\right)$

distinguished by the fact that they were not treated with insulin upon diagnosis. Furthermore, our study looked at a larger set of T1D and T2D loci, as well as comparing their roles in LADA against T1D and T2D, including taking population substructure into account. As with Howson et al. [23], we observed significant association of the T1D loci PTPN22, INS, HLA, and SH2B3. However, we did not observe significant association with the CLEC16A, IL2RA, CTLA4, and STAT loci. Despite published data observing the association of T2D locus TCF7L2 with a subset of T1D patients [24, 25], our study did not observe an association of this locus with LADA; one possibility could be that we used population-based controls, while previous studies may have used a different control strategy where the difference in the risk allele was more evident due to its under-representation in relatively disease-free controls. Our study goes further by leveraging GRS to offer a further line of evidence for the classification of diabetes subtypes, complementing standards for clinical decisionmaking and additional standardized (antibody) testing, each with their strengths and weaknesses.

LADA shows the MHC risk found in adult-onset T1D [23] with a reduced genetic susceptibility at this locus compared with childhood-onset T1D. Less clear is whether T2D loci play a role in adult-onset autoimmune diabetes. Our results show that genetic signals implicated in T1D or T2D both play a role in LADA, with four T1D loci and one T2D locus significantly associated with this form of diabetes. LADA is genetically more similar to T1D, especially when cases are constrained on both GADA+ and IA2A+, although LADA shares part of its genetic etiology with T2D. When constrained on GADA+ only, LADA cases became less distinct from T2D, highlighting the importance of IA2A in discriminating LADA within the T1D-T2D spectrum. By implication, a GRS derived from T1D can discriminate, to a degree, non-insulin requiring adult-onset diabetes patients with either autoimmune diabetes or T2D.

Regarding the loci implicated in T1D, our results are consistent with previous studies showing a major role for the MHC, PTPN22, and INS loci in LADA [10, 12, 13]. Interestingly, the risk allele frequency at INS (rs689) was even more strongly associated with LADA than with T1D. Therefore, our data strongly points to common insulin-related pathways underpinning autoimmune diabetes irrespective of the age at onset of the disease. Given the evidence that age at diagnosis is genetically determined [26], these loci may play a key role in determining the age at disease onset and the rate of disease progression.

While our results suggest LADA is genetically closer to T1D than to T2D, we observed an association at one T2D locus, HNF1A, known to be associated with T2D and 'maturity-onset diabetes of the young'; strikingly, the HNF1A signal remained significantly associated with LADA even in the cohort enriched for both T1D autoantibodies. Nevertheless, the nature of the role of HNF1A in LADA is unclear, although any gene compromising insulin secretory function could predispose to diabetes. This is the first report describing an association between this T2Dassociated risk allele and LADA, although this locus has been previously implicated in T1D [16]. Additionally, the strongest T2D-associated locus, TCF7L2, has been associated with LADA in a Finnish cohort [14-16], but in our study, the risk allele frequency in LADA was very close to that of controls and lower than controls in GADA+ IA2A + LADA. Our findings were further supported by leveraging healthy adult British controls from the WTCCC, which provided overall consistent results, including for the HNF1A signal. However, given the borderline association of T2D loci identified and the modest power in this single study, these signals must be subjected to replication efforts by independent investigators in order to fully validate these observations.

We found that, from GRS calculated from T1D- and T2D-implicated SNPs, which distinguished LADA cases from controls, the T1D GRS performed better than the T2D GRS; this difference was particularly striking in GADA+ IA2A + LADA cases. Comparison of GRS between the six defined groups placed LADA in between T1D and T2D but closer to T1D. GADA+ IA2A+ LADA was very similar to T1D, primarily because such constraint filters out 'T2D-like' cases and enriches for 'T1Dlike' cases. The potential for clinical, immunological, or genetic filters to define forms of diabetes is emphasized by the marked overlap in GRS scores, even between T1D and controls.

This study does have limitations. First, GADA-only LADA cases had a T2D-SNP GRS distribution more 
similar to T2D than controls. The specific association between the T2D risk score and GADA-only LADA cases could be in part due to the fact that a fraction of these cases might be false antibody-positive T2D, though those with double antibody positivity are likely to have a very low false positive rate. Thus, larger studies may resolve whether T2D risk alleles play a role in LADA. Indeed, this study was underpowered to identify specific associations other than for HNF1A. Second, two different genotyping arrays were utilized; thus, to correct for potential batch effects due to genotype array differences, population substructure, and relatedness among samples, we used a linear mixed model, resulting in highly conservative effect estimates. Consequently, it is possible that we have missed some true positive associations since we robustly controlled for false positive results.

The current nomenclature to classify diabetes, designating it as 'T1D' or 'T2D', was adopted to foster research and appropriate therapy for different phenotypic presentations. The combination of GRS, age at diagnosis, clinical phenotype, autoantibody assays, and C-peptide estimates as a proxy for insulin secretion affords a more sophisticated approach with the potential to dissect the heterogeneity of diabetes [6]. This study highlights the uncertainty of the current classification of diabetes [27]. These results suggest that clinical phenotype alone is insufficient to define the major types of diabetes. To better treat the various diabetes subtypes, we need to integrate the use of clinical phenotype, metabolic status, immune changes, and underlying genetic risk.

\section{Conclusion}

LADA is genetically closer to T1D than T2D, although the genetic load of T1D risk alleles is less than childhood-onset T1D, particularly at the MHC, potentially accounting for the later disease onset. Our results show that the genetic spectrum of T1D extends into adult-onset diabetes, where it can clinically masquerade as T2D. Furthermore, T2D genetic risk plays a small role in LADA, with a degree of evidence for the HNF1A locus, highlighting the potential for GRS to contribute towards defining diabetes subtypes.

\section{Additional file}

Additional file 1: Supplementary material. Table S1. Established SNPS for GRS. Table S2. Association of established T1D loci with LADA cases ( $P$-value LADA vs BMDCS) and comparison to T1D cases (LADA vs. T1D $P$-value). Table S3. Association of established T1D loci with GADA+ only LADA cases (P-value LADA vs BMDCS) and comparison to T1D cases (LADA vs. T1D P-value). Table S4. Association of established T1D loci with GADA+,IA2A+ LADA cases and comparison to T1D cases using controls. Table S5. Association of established T2D loci with LADA cases ( $P$-value LADA vs BMDCS) and comparison to T2D cases (LADA vs. T2D $P$-value). Table S6. Association of established T2D loci with GADA+ only LADA cases ( $P$-value LADA vs BMDCS) and comparison to T2D cases
(LADA vs. T2D P-value). Table S7. Association of established T2D loci with GADA+,IA2A+ LADA cases in comparison to association in T2D cases using controls. Supplementary Results. Follow-up analysis using WTCCC as an alternative control source. Table S8. Table of results for follow-up analysis using WTCCC as an alternative control source. Figure S1. Principle component analysis of LADA cases \& controls. Figure S2. Quantile-quantile plot of pruned markers used for PCs for LADA cases and controls. Figure S3. Extended set of ROC plots including Non-HLA and HLA only GRS also tested in LADA vs T1D and GADA+ only LADA vs GADA+,IA2A+ LADA. Weighted genetic risk scores (GRS) for T1D GRS (black), T1D without HLA GRS (blue) , HLA only GRS (cyan), T2D GRS (red) and both T1D and T2D GRS (green) were calculated and tested in A) LADA cases and controls B) GADA+ only and controls C) GADA+,IA2A+ LADA and controls D) LADA and WTCCC T1D cases E) GADA+ only LADA and WTCCC T1D F) GADA+,IA2A+ LADA and WTCCC T1D. Supplemental Methods. More details on recruitment of LADA cases and genotyping methods. (DOCX $1592 \mathrm{~kb}$ )

\section{Acknowledgements}

We would like to thank the JDRF, German Research Council (DFG: SFB 518, A1), German Diabetes Foundation, and EUFP5 (Action LADA) for providing samples for this research. This study makes use of data generated by the Wellcome Trust Case Control Consortium. A full list of the investigators who contributed to the generation of the data is available from www.wtccc.org.uk. Funding for the project was provided by the Wellcome Trust under award 076113.

The Bone Mineral Density in Childhood Study is a multicenter, longitudinal study of bone accrual in healthy children. Authors are listed below: Heidi J. Kalkwarf, PhD'; Joan M. Lappe, PhD²; Vicente Gilsanz, MD³; Sharon E. Oberfield, MD"; J John A. Shepherd, PhD ${ }^{5}$, Andrea Kelly, MD ${ }^{6,7}$, Babette S. Zemel, $\mathrm{PhD}^{6,8}$

'Division of Gastroenterology, Hepatology and Nutrition, Cincinnati Children's Hospital Medical Center, Cincinnati; ${ }^{2}$ Division of Endocrinology, Department of Medicine, Creighton University, Omaha; ${ }^{3}$ Department of Radiology, Children's Hospital Los Angeles, Los Angeles; ${ }^{4}$ Division of Pediatric Endocrinology, Diabetes, and Metabolism, Department of Pediatrics, Columbia University Medical Center, New York; ${ }^{5}$ Department of Radiology, University of California San Francisco, San Francisco; ${ }^{6}$ Department of Pediatrics, Perelman School of Medicine, University of Pennsylvania, Philadelphia; 'Division of Endocrinology and Diabetes, The Children's Hospital of Philadelphia, Philadelphia; ${ }^{8}$ Division of Gastroenterology, Hepatology and Nutrition, The Children's Hospital of Philadelphia, Philadelphia.

\section{Funding}

SFAG is supported by the NIH (R01 DK085212) and the Daniel B. Burke Endowed Chair for Diabetes Research. DM is supported by CIBERDEM, Instituto de Salud Carlos III (Spain).

\section{Availability of data and material}

The datasets generated and/or analyzed during the current study are not publicly available until the completion of R01 DK085212, but are available from the corresponding author on reasonable request.

\section{Authors' contributions}

Study concept and design: RM, AC, DLC, MIH, JPB, KMH, VCG, HH, DM, NCS, $\mathrm{KBY}, \mathrm{BFV}, \mathrm{SS}, \mathrm{BOB}, \mathrm{RDL}$, and SFAG. Analysis and interpretation of data: RM, $A C, D L C, J P B, B F V$, and SFAG. Resources: MIH, HH, DM, NCS, KBY, BFV, SS, $B O B, R D L$, and SFAG. Drafting and critical revision of the manuscript: RM, AC, DLC, BOB, RDL, and SFAG. Obtained funding: SS, BOB, RDL, and SFAG. All authors contributed to the final version of the manuscript. RM, AC, DLC, BOB, $R D L$, and SFAG are the guarantors of this work and, as such, had full access to all the data in the study and take responsibility for the integrity of the data and the accuracy of the data analysis. All authors read and approved the final manuscript.

\section{Authors' information}

Nanette C. Schloot is on leave of absence from the German Diabetes Center and is currently employed by Lilly Deutschland, Bad Homburg, Germany. Vanessa C. Guy is currently employed by Science 37 Inc. 


\section{Competing interests}

The authors declare that they have no competing interests.

\section{Consent for publication}

Not applicable.

\section{Ethics approval and consent to participate}

The study was approved by the UK National Research Ethics Committee as well as the University Medical Center Ulm, Hospital Universitari Germans Trias i Pujol, the German Diabetes Center in Düsseldorf, Odense University Hospital, and the Children's Hospital of Philadelphia.

\section{Publisher's Note}

Springer Nature remains neutral with regard to jurisdictional claims in published maps and institutional affiliations.

\section{Author details}

'Division of Human Genetics, The Children's Hospital of Philadelphia, Philadelphia, PA, USA. ${ }^{2}$ Department of Genetics, Perelman School of Medicine, University of Pennsylvania, Philadelphia, PA, USA. ${ }^{3}$ Department of Immunobiology, Barts and the London School of Medicine and Dentistry, Queen Mary University of London, London, UK. ${ }^{4}$ Center for Applied Genomics, The Children's Hospital of Philadelphia, Philadelphia, PA, USA ${ }^{5}$ Department of Pediatrics, Perelman School of Medicine, University of Pennsylvania, Philadelphia, PA, USA. ${ }^{6}$ Hospital Universitari Germans Trias i Pujol, Barcelona, Spain. ${ }^{7}$ German Diabetes Center, Düsseldorf, Germany. ${ }^{8}$ Odense University Hospital, Odense, Denmark. ${ }^{9}$ Department of Systems Pharmacology and Translational Therapeutics, Perelman School of Medicine, University of Pennsylvania, Philadelphia, PA, USA. ${ }^{10}$ Main Line Health System, Wynnewood, PA, USA. ${ }^{11}$ Department of Internal Medicine I, Ulm University Medical Centre, Ulm, Germany. ${ }^{12}$ LKC School of Medicine, Nanyang Technological University, Singapore and Imperial College, London, UK. ${ }^{13}$ Divisions of Human Genetics and Endocrinology, The Children's Hospital of Philadelphia, 3615 Civic Center Boulevard, Room 1102D, Philadelphia, PA 19104, USA. ${ }^{14}$ Department of Immunobiology, Blizard Institute, 4 Newark Street, London E1 2AT, UK

Received: 4 January 2017 Accepted: 29 March 2017

Published online: 25 April 2017

\section{References}

1. Maddaloni E, Lessan N, Al Tikriti A, Buzzetti R, Pozzilli P, Barakat MT. Latent autoimmune diabetes in adults in the United Arab Emirates: clinical features and factors related to insulin-requirement. PLoS One. 2015;10(8), e0131837.

2. Hawa Ml, Kolb H, Schloot N, Beyan H, Paschou SA, Buzzetti R, Mauricio D, De Leiva A, Yderstraede $\mathrm{K}$, Beck-Neilsen $\mathrm{H}$, et al. Adult-onset autoimmune diabetes in Europe is prevalent with a broad clinical phenotype: Action LADA 7. Diabetes Care. 2013:36(4):908-13.

3. Tuomi T, Carlsson A, Li H, Isomaa B, Miettinen A, Nilsson A, Nissen M, Ehrnstrom BO, Forsen B, Snickars B, et al. Clinical and genetic characteristics of type 2 diabetes with and without GAD antibodies. Diabetes. 1999;48(1): $150-7$.

4. Zhou Z, Xiang Y, Ji L, Jia W, Ning G, Huang G, Yang L, Lin J, Liu Z, Hagopian WA, et al. Frequency, immunogenetics, and clinical characteristics of latent autoimmune diabetes in China (LADA China study): a nationwide, multicenter, clinic-based cross-sectional study. Diabetes. 2013;62(2):543-50.

5. Tuomi T, Groop LC, Zimmet PZ, Rowley MJ, Knowles W, Mackay IR. Antibodies to glutamic acid decarboxylase reveal latent autoimmune diabetes mellitus in adults with a non-insulin-dependent onset of disease. Diabetes. 1993;42(2):359-62.

6. Oram RA, Patel K, Hill A, Shields B, McDonald TJ, Jones A, Hattersley AT, Weedon MN. A Type 1 diabetes genetic risk score can aid discrimination between type 1 and type 2 diabetes in young adults. Diabetes Care. 2016; 39(3):337-44

7. Bradfield JP, Qu HQ, Wang K, Zhang H, Sleiman PM, Kim CE, Mentch FD, Qiu $\mathrm{H}$, Glessner JT, Thomas KA, et al. A genome-wide meta-analysis of six type 1 diabetes cohorts identifies multiple associated loci. PLoS Genet. 2011;7(9), e1002293.

8. Wellcome Trust Case Control C. Genome-wide association study of 14,000 cases of seven common diseases and 3,000 shared controls. Nature. 2007; 447(7145):661-78
9. Consortium DIAGRM-a, Consortium AGENTD, Consortium SATD, Consortium MATD, Consortium TDGEbN-gsim-ES, Mahajan A, Go MJ, Zhang W, Below JE, Gaulton KJ, et al. Genome-wide trans-ancestry meta-analysis provides insight into the genetic architecture of type 2 diabetes susceptibility. Nat Genet. 2014;46(3):234-44.

10. Desai M, Zeggini E, Horton VA, Owen KR, Hattersley AT, Levy JC, Walker M, Gillespie KM, Bingley PJ, Hitman GA, et al. An association analysis of the HLA gene region in latent autoimmune diabetes in adults. Diabetologia. 2007; 50(1):68-73.

11. Horton V, Stratton I, Bottazzo GF, Shattock M, Mackay I, Zimmet P, Manley S, Holman R, Turner R. Genetic heterogeneity of autoimmune diabetes: age of presentation in adults is influenced by HLA DRB1 and DQB1 genotypes (UKPDS 43). UK Prospective Diabetes Study (UKPDS) Group. Diabetologia. 1999;42(5):608-16

12. Cervin C, Lyssenko V, Bakhtadze E, Lindholm E, Nilsson P, Tuomi T, Cilio CM, Groop L. Genetic similarities between latent autoimmune diabetes in adults, type 1 diabetes, and type 2 diabetes. Diabetes. 2008;57(5):1433-7.

13. Desai M, Zeggini E, Horton VA, Owen KR, Hattersley AT, Levy JC, Hitman GA, Walker M, Holman RR, McCarthy Ml, et al. The variable number of tandem repeats upstream of the insulin gene is a susceptibility locus for latent autoimmune diabetes in adults. Diabetes. 2006;55(6):1890-4.

14. Lukacs K, Hosszufalusi N, Dinya E, Bakacs M, Madacsy L, Panczel P. The type 2 diabetes-associated variant in TCF7L2 is associated with latent autoimmune diabetes in adult Europeans and the gene effect is modified by obesity: a meta-analysis and an individual study. Diabetologia. 2012;55(3): 689-93.

15. Zampetti S, Spoletini M, Petrone A, Capizzi M, Arpi ML, Tiberti C, Di Pietro S, Bosi E, Pozzilli P, Giorgino F, et al. Association of TCF7L2 gene variants with low GAD autoantibody titre in LADA subjects (NIRAD Study 5). Diabet Med. 2010;27(6):701-4.

16. Andersen MK, Sterner M, Forsen T, Karajamaki A, Rolandsson O, Forsblom C, Groop PH, Lahti K, Nilsson PM, Groop L, et al. Type 2 diabetes susceptibility gene variants predispose to adult-onset autoimmune diabetes. Diabetologia. 2014;57(9):1859-68.

17. Kalkwarf HJ, Zemel BS, Gilsanz V, Lappe JM, Horlick M, Oberfield S, Mahboubi S, Fan B, Frederick MM, Winer K, et al. The bone mineral density in childhood study: bone mineral content and density according to age, sex, and race. J Clin Endocrinol Metab. 2007;92(6):2087-99.

18. Zhou X, Stephens M. Genome-wide efficient mixed-model analysis for association studies. Nat Genet. 2012;44(7):821-4.

19. Cho YS, Chen CH, Hu C, Long J, Ong RT, Sim X, Takeuchi F, Wu Y, Go MJ, Yamauchi $T$, et al. Meta-analysis of genome-wide association studies identifies eight new loci for type 2 diabetes in east Asians. Nat Genet. 2012; 44(1):67-72

20. Winkler C, Krumsiek J, Buettner F, Angermuller C, Giannopoulou EZ, Theis FJ, Ziegler AG, Bonifacio E. Feature ranking of type 1 diabetes susceptibility genes improves prediction of type 1 diabetes. Diabetologia. 2014;57(12) 2521-9.

21. Kundu S, Aulchenko YS, van Duijn CM, Janssens AC. PredictABEL: an R package for the assessment of risk prediction models. Eur J Epidemiol. 2011; 26(4):261-4.

22. Leslie RD, Palmer J, Schloot NC, Lernmark A. Diabetes at the crossroads: relevance of disease classification to pathophysiology and treatment. Diabetologia. 2016:59(1):13-20.

23. Howson JM, Rosinger S, Smyth DJ, Boehm BO, Group A-ES, Todd JA. Genetic analysis of adult-onset autoimmune diabetes. Diabetes. 2011;60(10): 2645-53.

24. Redondo MJ, Grant SF, Davis A, Greenbaum C, Biobank TDE. Dissecting heterogeneity in paediatric Type 1 diabetes: association of TCF7L2 rs7903146 TT and low-risk human leukocyte antigen (HLA) genotypes. Diabet Med. 2017:34(2):286-90.

25. Redondo MJ, Muniz J, Rodriguez LM, lyer D, Vaziri-Sani F, Haymond MW, Hampe CS, Metzker ML, Grant SF, Balasubramanyam A. Association of TCF7L2 variation with single islet autoantibody expression in children with type 1 diabetes. BMJ Open Diabetes Res Care. 2014;2(1), e000008.

26. Fava D, Gardner S, Pyke D, Leslie RD. Evidence that the age at diagnosis of IDDM is genetically determined. Diabetes Care. 1998;21(6):925-9.

27. Schwartz SS, Epstein S, Corkey BE, Grant SF, Gavin 3rd JR, Aguilar RB. The time is right for a new classification system for diabetes: rationale and implications of the beta-cell-centric classification schema. Diabetes Care. 2016;39(2):179-86. 